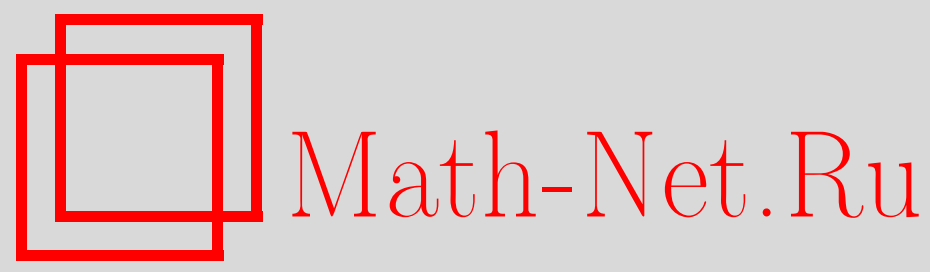

С. А. Теляковский, О скорости сходимости рядов Фурье функций ограниченной вариации, Матем. заметки, 2002, том 72, выпуск 6, 949-953

DOI: https://doi.org/10.4213/mzm677

Использование Общероссийского математического портала Math-Net.Ru подразумевает, что вы прочитали и согласны с пользовательским соглашением http://www.mathnet.ru/rus/agreement

Параметры загрузки:

IP: 18.234 .156 .22

26 апреля 2023 г., $12: 32: 16$ 


\section{О СКОРОСТИ СХОДИМОСТИ РЯДОВ ФУРЬЕ ФУНКЦИЙ ОГРАНИЧЕННОЙ ВАРИАЦИИ}

\section{С. А. Теляковский}

Пусть $2 \pi$-периодическая функция $f$ имеет ограниченную вариацию,

$$
\frac{a_{0}}{2}+\sum_{k=1}^{\infty}\left(a_{k} \cos k x+b_{k} \sin k x\right)
$$

- ее ряд Фурье, $s_{n}(f, x)$ - частная сумма порядка $n$ ряда (1) и для всех $x$ вьполняется условие

$$
f(x)=\frac{1}{2}(f(x+0)+f(x-0)) .
$$

Введем обозначения, которые будут испольоваться в дальнйшем:

$$
g_{x}(t):=f(x+t)+f(x-t)-2 f(x), \quad t \in[0, \pi],
$$

$V(f,[\alpha, \beta])$ - вариация функции $f$ на отрезке $[\alpha, \beta], V(f)$ - полная вариация функции $f$ на периоде. Через $C$ будут обозначаться абсолютные положительные постоянные, в разных случаях различные.

В силу (2) функция $g_{x}(t)$ непрерьвна в нуле, поэтому

$$
V\left(g_{x},[0, t]\right) \rightarrow 0, \quad t \rightarrow 0 .
$$

Согласно теореме Дирихле-Жордана в каждой точке $x$ ряд Фурье функции $f$ сходится к значению $f(x)$, т.е.

$$
f(x)-s_{n}(f, x) \rightarrow 0, \quad n \rightarrow \infty .
$$

Боянич [1] установил следующую оценку скорости сходимости в (4):

$$
\left|f(x)-s_{n}(f, x)\right| \leqslant \frac{3}{n} \sum_{k=1}^{n} V\left(g_{x},\left[0, \frac{\pi}{k}\right]\right) .
$$

Заметим, что в силу (3) выражение в правой части неравенства (5) стремится к нулю при $n \rightarrow \infty$.

Пусть $n_{1}=1<n_{2}<\cdots$-последовательность натуральных чисел, удовлетворяющая условию

$$
\sum_{j=m}^{\infty} \frac{1}{n_{j}} \leqslant \frac{A}{n_{m}}, \quad m=1,2, \ldots,
$$

где $A>1$ - некоторая постоянная. Это условие равносильно представимости последовательности $\left\{n_{j}\right\}$ в виде объединения конечного числа лакунарных последовательностей (cм. [2, с. 24]). Некоторые другие условия, равносильные (6), были получены в [3, лемма 1$]$.

В [4, теорема 2] установлен следующий резултат, усиливающий соотношение (4). Если функция $f$ имеет ограниченную вариацию и последовательность $\left\{n_{j}\right\}$ удовлетворяет условию (6), то ряд

$$
\sum_{j=1}^{\infty}\left|\sum_{k=n_{j}}^{n_{j+1}-1}\left(a_{k} \cos k x+b_{k} \sin k x\right)\right|
$$

сходится в каждой точке $x$ и его сумма ограничена числом $C A V(f)$, где $A$ - множитель из оценки (6).

В [5] показано, что условие (6) на последовательность $\left\{n_{j}\right\}$ не является в этом утверждении необходимым, но вместе с тем, оно не может быть существенно ослаблено.

Цель настоящей работы - показать, что усиление, аналогичное (7), допускает и оценка (5).

Работа выполнена при финансовой поддержке Российского фонда фундаментальных исследований, грант № 99-01-01210, и программ “Ведущие научные школы”, грант № 00-15-96047, и INTAS, грант № 99-01080. 
ТЕОРема 1. Если функиия $f$ имеет ограниченную вариачию и последовательность $\left\{n_{j}\right\}$ удовлетворяет условию (6), то для всех $n$ и х справедлива оченка

$$
\begin{aligned}
\left|f(x)-s_{n}(f, x)\right| & =\left|\sum_{k=n+1}^{\infty}\left(a_{k} \cos k x+b_{k} \sin k x\right)\right| \\
& \leqslant\left|\sum_{k=n+1}^{n_{i}-1}\left(a_{k} \cos k x+b_{k} \sin k x\right)\right|+\sum_{j=i}^{\infty}\left|\sum_{k=n_{j}}^{n_{j+1}-1}\left(a_{k} \cos k x+b_{k} \sin k x\right)\right| \\
& \leqslant \frac{C A}{n+1} \sum_{k=1}^{n+1} V\left(g_{x},\left[0, \frac{\pi}{k}\right]\right)
\end{aligned}
$$

где $n_{i-1} \leqslant n<n_{i}$ и $A$ - множитель из оченки (6).

ДокАЗАТЕЛЬСтво. Сначала оценим остаток ряда (7). Так как

$$
\begin{aligned}
\sum_{k=n_{j}}^{n_{j+1}-1}\left(a_{k} \cos k x+b_{k} \sin k x\right) & =\frac{1}{\pi} \int_{0}^{\pi} g_{x}(t) \sum_{k=n_{j}}^{n_{j+1}-1} \cos k t d t \\
& =\frac{1}{\pi} \int_{0}^{\pi} g_{x}(t) d \sum_{k=n_{j}}^{n_{j+1}-1} \frac{\sin k t}{k}=\frac{1}{\pi} \int_{0}^{\pi} \sum_{k=n_{j}}^{n_{j+1}-1} \frac{\sin k t}{k} d g_{x}(t)
\end{aligned}
$$

To

$$
\left|\sum_{k=n_{j}}^{n_{j+1}-1}\left(a_{k} \cos k x+b_{k} \sin k x\right)\right| \leqslant \frac{1}{\pi} \int_{0}^{\pi}\left|\sum_{k=n_{j}}^{n_{j+1}-1} \frac{\sin k t}{k}\right| d V\left(g_{x},[0, t]\right)
$$

и, значит,

$$
\sum_{j=i}^{\infty}\left|\sum_{k=n_{j}}^{n_{j+1}-1}\left(a_{k} \cos k x+b_{k} \sin k x\right)\right| \leqslant \frac{1}{\pi} \int_{0}^{\pi} \sum_{j=i}^{\infty}\left|\sum_{k=n_{j}}^{n_{j+1}-1} \frac{\sin k t}{k}\right| d V\left(g_{x},[0, t]\right) .
$$

Разобьем промежуток интегрирования в интеграле из (9) на два отрезка: $\left[0, \pi / n_{i}\right]$ и $\left[\pi / n_{i}, \pi\right]$. Согласно теореме 1 из [4] справедлива равномерная по $t$ оценка

$$
\sum_{j=1}^{\infty}\left|\sum_{k=n_{j}}^{n_{j+1}-1} \frac{\sin k t}{k}\right| \leqslant C A
$$

С ее помощью получаем

$$
\int_{0}^{\pi / n_{i}} \sum_{j=i}^{\infty}\left|\sum_{k=n_{j}}^{n_{j+1}-1} \frac{\sin k t}{k}\right| d V\left(g_{x},[0, t]\right) \leqslant C A V\left(g_{x},\left[0, \frac{\pi}{n_{i}}\right]\right) .
$$

Далее, в [6, лемма 1] фактически доказана оценка

$$
\sum_{j=i}^{\infty}\left|\sum_{k=n_{j}}^{n_{j+1}-1} \frac{\sin k t}{k}\right| \leqslant \frac{C A}{n_{i} t}, \quad 0<t \leqslant \pi
$$


Пользуясь этой оценкой, находим

$$
\int_{\pi / n_{i}}^{\pi} \sum_{j=i}^{\infty}\left|\sum_{k=n_{j}}^{n_{j+1}-1} \frac{\sin k t}{k}\right| d V\left(g_{x},[0, t]\right) \leqslant \frac{C A}{n_{i}} \int_{\pi / n_{i}}^{\pi} \frac{1}{t} d V\left(g_{x},[0, t]\right)
$$

Для оценки интеграла из правой части неравенства (11) повторяем соответствующие рассуждения из работы [1]:

$$
\begin{aligned}
\int_{\pi / n_{i}}^{\pi} \frac{1}{t} d V\left(g_{x},[0, t]\right) & =\left.\frac{1}{t} V\left(g_{x},[0, t]\right)\right|_{\pi / n_{i}} ^{\pi}+\int_{\pi / n_{i}}^{\pi} V\left(g_{x},[0, t]\right) \frac{d t}{t^{2}} \\
& \leqslant \frac{1}{\pi} V\left(g_{x},[0, \pi]\right)+\frac{1}{\pi} \int_{1}^{n_{i}} V\left(g_{x},\left[0, \frac{\pi}{t}\right]\right) d t \\
& \leqslant \frac{2}{\pi} \sum_{k=1}^{n_{i}-1} V\left(g_{x},\left[0, \frac{\pi}{k}\right]\right) .
\end{aligned}
$$

Из (9)-(12) вытекает следующая оценка остатка ряда (7):

$$
\sum_{j=i}^{\infty}\left|\sum_{k=n_{j}}^{n_{j+1}-1}\left(a_{k} \cos k x+b_{k} \sin k x\right)\right| \leqslant \frac{C A}{n_{i}} \sum_{k=1}^{n_{i}} V\left(g_{x},\left[0, \frac{\pi}{k}\right]\right) .
$$

Если число $n+1$ не входит в последовательность $\left\{n_{j}\right\}$, то, чтобы оценить выражение

$$
\left|\sum_{k=n+1}^{n_{i}-1}\left(a_{k} \cos k x+b_{k} \sin k x\right)\right|+\sum_{j=i}^{\infty}\left|\sum_{k=n_{j}}^{n_{j+1}-1}\left(a_{k} \cos k x+b_{k} \sin k x\right)\right|
$$

добавим в последовательность $\left\{n_{j}\right\}$ число $n+1$. Так как для полученной новой последовательности выполняется условие вида (6), если заменить в нем $A$ на $2 A$, это позволяет в силу (13) оценить выражение (14) суммой

$$
\frac{C A}{n+1} \sum_{k=1}^{n+1} V\left(g_{x},\left[0, \frac{\pi}{k}\right]\right)
$$

Теорема доказана.

Далее будем предполагать, что функция $f$ не только имеет ограниченную вариацию, но и удовлетворяет некоторым условиям непрерьвности.

Как отмечено в [1], если функция $f$ непрерывна всюду, то

$$
\left.V\left(g_{x},[0, \delta]\right) \leqslant V(f,[x-\delta, x+\delta]) \leqslant 2 \omega(V(f,[-\pi, t]), \delta)\right),
$$

где $\omega(V(f,[-\pi, t]), \delta))$ - модуль непрерывности функции $V(f,[-\pi, t])$. Поэтому из $(5)$ следует оценка

$$
\left|f(x)-s_{n}(f, x)\right| \leqslant \frac{6}{n} \sum_{k=1}^{n} \omega\left(V(f,[-\pi, t]), \frac{\pi}{k}\right),
$$

которая эквивалентна (с точностью до числового множителя) результату, полученному НатансоHом [7]. 
А из (8) в силу (15) получаем следующее усиление оценки (16):

$$
\begin{aligned}
& \left|\sum_{k=n+1}^{n_{i}-1}\left(a_{k} \cos k x+b_{k} \sin k x\right)\right|+\sum_{j=i}^{\infty}\left|\sum_{k=n_{j}}^{n_{j+1}-1}\left(a_{k} \cos k x+b_{k} \sin k x\right)\right| \\
& \leqslant \frac{C A}{n+1} \sum_{k=1}^{n+1} \omega\left(V(f,[-\pi, t]), \frac{\pi}{k}\right), \quad n_{i-1} \leqslant n<n_{i} .
\end{aligned}
$$

Рассмотрим теперь, что можно сказать, если функция ограниченной вариации непрерывна в некоторой точке или на части периода.

В [6, теорема 3] доказано, что если функция $f$ имеет ограниченную вариацию и, кроме того, непрерьвна в точке $x_{0}$, то ряд (7) сходится в этой точке равномерно. А если $f$ непрерьвна во всех точках отрезка $[\alpha, \beta]$, то ряд (7) сходится равномерно на $[\alpha, \beta]$.

Оба эти утверждения можно вывести из оценки (8).

В самом деле, если функция $f(x)$ непрерьвна в точке $x_{0}$, то в этой точке непрерьвна также функция $V(f,[-\pi, x])$. Поэтому по заданному $\varepsilon>0$ можно найти положительное $\delta$, для которого выполняется оценка

$$
V\left(f,\left[x_{0}-2 \delta, x_{0}+2 \delta\right]\right)<\varepsilon .
$$

Пусть $N$ таково, что $\pi / N \leqslant \delta$. Тогда для всех $k>N$ и $x$ таких, что $\left|x-x_{0}\right| \leqslant \delta$, имеем

$$
V\left(g_{x},\left[0, \frac{\pi}{k}\right]\right) \leqslant V\left(f,\left[x-\frac{\pi}{k}, x+\frac{\pi}{k}\right]\right)<\varepsilon .
$$

Значит, согласно (8) для этих $x$ и $n_{i}>N$ вьполняется оценка

$$
\sum_{j=i}^{\infty}\left|\sum_{k=n_{j}}^{n_{j}+1}\left(a_{k} \cos k x+b_{k} \sin k x\right)\right|<\frac{C A}{n_{i}}\left(\sum_{k=1}^{N} V\left(g_{x},\left[0, \frac{\pi}{k}\right]\right)+\sum_{k=N+1}^{n_{i}} \varepsilon\right)<\frac{C A}{n_{i}} N V(f)+C A \varepsilon
$$

Так как за счет выбора числа $i$ величину

$$
\frac{C A}{n_{i}} N V(f)
$$

можно сделать сколь угодно малой, то из (18) вытекает равномерная сходимость ряда (7) в точке $x_{0}$.

Подобными рассуждениями устанавливается и равномерная сходимость ряда (7) на отрезке $[\alpha, \beta]$, если функция $f$ непрерывна во всех точках этого отрезка. Будем считать, что отрезок $[\alpha, \beta]$ принадлежит интервалу $(-\pi, \pi)$.

Действительно, вместе с $f(x)$ функция $V(f,[-\pi, x])$ непрерывна во всех точках отрезка $[\alpha, \beta]$. Пользуясь равномерной непрерьвностью функции $V(f,[-\pi, x])$ на $[\alpha, \beta]$ и ее непрерьвностью в точках $\alpha$ и $\beta$, видим, что по заданному $\varepsilon>0$ можно найти $\delta>0$ такое, что для всех $x \in[\alpha, \beta]$

$$
V(f,[x-\delta, x+\delta])<\varepsilon .
$$

Поэтому если $\pi / N<\delta$, то для $k>N$ и всех $x \in[\alpha, \beta]$ справедлива оценка

$$
V\left(g_{x},\left[0, \frac{\pi}{k}\right]\right) \leqslant V\left(f,\left[x-\frac{\pi}{k}, x+\frac{\pi}{k}\right]\right)<\varepsilon .
$$

Значит, если $n_{i}>N$, то в силу (8) для всех $x \in[\alpha, \beta]$ справедлива оценка (18), из которой следует равномерная на $[\alpha, \beta]$ сходимость ряда $(7)$.

В заключение отметим, что если функция $f$ непрерывна на отрезке $[\alpha, \beta]$, то из (8) можно получить оценку скорости равномерной сходимости ряда (7) на отрезках вида $[\alpha+\delta, \beta-\delta], \delta>0$. 
ТЕОРема 2. Пусть функиия $f$ имеет ограниченную вариацию на периоде и непрерывна на отрезке $[\alpha, \beta]$, а последовательность $\left\{n_{j}\right\}$ удовлетворяет условию (6). Если $0<\delta<(\beta-\alpha) / 2$, то для всех $x \in[\alpha+\delta, \beta-\delta]$ и $n_{i}>[\pi / \delta]$ справедлива оченка

$$
\begin{aligned}
\left|f(x)-s_{n_{i}-1}(f, x)\right| & \leqslant \sum_{j=i}^{\infty}\left|\sum_{k=n_{j}}^{n_{j+1}-1}\left(a_{k} \cos k x+b_{k} \sin k x\right)\right| \\
& \leqslant \frac{C A}{n_{i}}\left(\sum_{k=[\pi / \delta]+1}^{n_{i}} \omega\left(V(f,[\alpha, x]), \frac{\pi}{k}\right)+\frac{V(f)}{\delta}\right),
\end{aligned}
$$

где $\omega(V(f,[\alpha, x]))$ - модуль непрерывности функиии $V(f,[\alpha, x])$ как функции, заданной на $[\alpha, \beta]$.

В самом деле, для $x \in[\alpha+\delta, \beta-\delta]$ при $k>[\pi / \delta]$ имеем

$$
V\left(g_{x}, \frac{\pi}{k}\right) \leqslant 2 \omega\left(V(f,[\alpha, x]), \frac{\pi}{k}\right) .
$$

Поэтому для этих $x$ и $n_{i}>[\pi / \delta]$ оценка (19) вытекает из (8).

\section{СПИСОК ЦИТИРОВАННОЙ ЛИТЕРАТУРЫ}

1. Bojanić R. // Publications de l'institut mathématique (Beograd). 1979. T. 26(40). C. 57-60. 2. Бари Н. К. Тригонометрические ряды. М.: Физматгиз, 1961. 3. Стечкин С. Б. // Изв. АН СССР. Сер. матем. 1956. Т. 20. С. 385-412. 4. Теляковский С. А. // Тр. МИАН. 1997. Т. 219. С. 378-386. 5. Попов А. Ю., Теляковский С. А. // Изв. вузов. Матем. 2000. Т. 1. С. 51-55. 6. Теляковский С. А. // Тр. МИАН. 2001. Т. 232. С. 318-326. 7. Натансон Г. И. // Вестн. ЛГУ. Матем., мех., астрон. 1972. Т. 7. С. 154-155. 